\title{
Electron Microscopy of Probability Currents at Atomic Resolution
}

\author{
A. Lubk, ${ }^{1}$ A. Béché, ${ }^{2}$ and J. Verbeeck ${ }^{2}$ \\ ${ }^{1}$ Triebenberg Laboratory, Institute of Structure Physics, Technische Universität Dresden, 01062 Dresden, Germany \\ ${ }^{2}$ EMAT, University of Antwerp, Groenenborgerlaan 171, 2020 Antwerp, Belgium
}

(Received 10 July 2015; published 20 October 2015)

\begin{abstract}
Atomic resolution transmission electron microscopy records the spatially resolved scattered electron density to infer positions, density, and species of atoms. These data are indispensable for studying the relation between structure and properties in solids. Here, we show how this signal can be augmented by the lateral probability current of the scattered electrons in the object plane at similar resolutions and fields of view. The currents are reconstructed from a series of three atomic resolution TEM images recorded under a slight difference of perpendicular line foci. The technique does not rely on the coherence of the electron beam and can be used to reveal electric, magnetic, and strain fields with incoherent electron beams as well as correlations in inelastic transitions, such as electron magnetic chiral dichroism.
\end{abstract}

DOI: 10.1103/PhysRevLett.115.176101

Transmission electron microscopy (TEM) owes its power and versatility as a characterization tool in materials science to a combination of high (atomic) resolution and strong interaction of the beam electrons with the target. Here, a considerable number of specimen properties show themselves in a local modulation of the electron's scattering direction rather than the intensity in the object plane. One important example is long range electric and magnetic fields, e.g., due to drift-diffusion potentials or magnetic domains, which change the electron direction by virtue of the Lorentz force. A second one is strained crystal lattices, such as employed in electronic devices, which vary the scattering direction within a diffracted beam. A third example is chiral inelastic interactions, responsible for, e.g., the electron magnetic chiral dichroism (EMCD) [1], changing the angular momentum of the beam. To probe these important specimen characteristics a number of ingenious indirect imaging modi have been invented. Off-axis electron holography (EH) [2] permits the reconstruction of the phase of a coherent electron beam, the gradient of which is proportional to the local scattering direction in the semiclassic limit. A plethora of EH studies on electric, magnetic, and strain fields have revealed a wealth of information such as the structure of an extended Skyrmion lattice [3] or long range strain distributions in strain engineered MOSFETs [4,5]. In the case of inelastic transitions the local anisotropy of scattering directions as imposed by EMCD has been probed by a combination of scanning TEM (STEM) [6] and anisotropic probe [7] or detector shapes [8]. In spite of the remarkable success of these methods, there exists no unique setup capable of providing all the above results directly. Furthermore, the above indirect methods are limited in certain aspects (e.g., coherency for $\mathrm{EH}$, atomic resolution for EMCD), rendering alternatives highly valuable.

Here, we tackle the problem by setting up a novel TEM mode capable of reconstructing the 2D lateral quantum
PACS numbers: 68.37.Lp, 03.65.Nk, 41.85.-p, 61.05.J-

mechanical probability current $\mathbf{j}(\mathbf{r})$ of the scattered electrons in the image plane (coordinates $\mathbf{r}$ ) over large fields of view down to atomic resolution. The probability current is the local measure of the scattering direction of the electrons [9], therefore providing information on the target properties discussed above (see below for detailed relations). By virtue of these relations a large number of solid state properties can be studied largely irrespective of the coherency of the electron beam because $\mathbf{j}$ retains its meaning for incoherent electrons. The latter distinguishes this TEM mode from EH, which relies on coherent illumination. Consequently, measuring $\mathbf{j}$ can vastly generalize the scope of TEM studies in regimes, where low coherent signals are intrinsic to the imaging mode, such as in ultrafast TEM [10-12] or energy-loss filtered TEM imaging. The former employs short bunches of electrons generated by photoelectric emission from a laser shining on a cathode. Here, the size of the incoherently emitting area is increased with respect to standard Schottky or field emission tips. In energy filtered TEM (EFTEM) on the other hand, the inelastic interaction falling within the energy range selected by the filter removes a large part of the phase relationship within the beam.

The proposed method has a number of advantages and disadvantages compared to its scanning TEM counterpart referred to as STEM differential phase contrast (DPC) $[13,14]$. It can be shown that the latter records the integral of the probe's probability current per scanning point [15]. STEM-DPC has been used extensively for medium resolution magnetic and electric field studies and was recently extended to atomic resolution $[16,17]$. However, STEM-DPC suffers from scanning errors [17] and requires dedicated detectors with a small number of pixels and high read-out speed [18]. This puts restrictions on the field of view, the accuracy of the measured probability current, and the flexibility to use the method in combination with other 
TEM techniques such as EFTEM. We show below, that the TEM scheme removes some of these issues, e.g., by providing an undistorted probability current over large fields of view. The price to be paid is a delicate (but feasible) adjustment of line foci and an additional regularization required to suppress the amplification of noise in the reconstruction.

We proceed with a short discussion on the physical significance of the lateral probability current, before illustrating the theoretical and experimental principles of measuring the probability current down to atomic resolution in the TEM. The 2D lateral probability current of the scattered electrons in the object exit plane may be both spectrally resolved (energy loss $\delta E$ ) and decomposed into a conservative (curl-free) and solenoidal current by virtue of the Helmholtz decomposition $\mathbf{j}(\delta E)=\mathbf{j}_{\mathrm{c}}(\delta E)+\mathbf{j}_{\mathrm{r}}(\delta E)$ [19]. Note that the work by Paganin and Nugent [19] elaborates on a different quantity than the one treated here: while in Ref. [19] the Pointing vector of electromagnetic radiation divided by the intensity is considered, we evaluate the probability current of electrons. Depending on the specimen interaction and energy loss regime considered, one can identify several particularly simple approximate links between the current density and physical properties of the target.

(i) Within the scope of the phase object approximation, valid for weakly scattering targets (e.g., soft matter) and/or medium resolution, the probability current is proportional to the projected lateral electric $\left(E_{x, y}\right)$ and magnetic field $\left(B_{x, y}\right)$ according to $\mathbf{j} \sim \int\left[-(1 / v)\left(E_{x}, E_{y}\right)^{T}+\left(B_{y},-B_{x}\right)^{T}\right] d z$ [15]. Here $v$ is the velocity of the incoming electrons and we employed Hartree atomic units $\left(m_{0}=\hbar=e=1\right)$.

(ii) The dark field current within some systematic beam g diffracted from a strained crystal is proportional to the gradient of the displacement field $\mathbf{u}(\cong$ elements of the strain tensor): $\mathbf{j}_{\mathbf{g}} \sim-\nabla(\mathbf{g} \cdot \mathbf{u})$.

(iii) Employing illumination conditions (with lateral incident momentum $\mathbf{k}$ ) predominantly represented by a single Bloch wave (Bloch vector $\boldsymbol{\gamma}=\mathbf{k}$ ) within the crystal, the current averaged over one unit cell with surface area $\Omega$ in the object exit plane is proportional to the gradient of the Bloch band: $\overline{\mathbf{j}}=\int_{\Omega} \mathbf{j}(\mathbf{r}) d^{2} r=-\nabla_{\gamma} q_{z}(\mathbf{k})$ [20].

(iv) The current pertaining to beam electrons, which have excited a dipole allowed excitation of a core electron in the target, is linked to the difference probability of absorbing a virtual photon of left or right circular polarization. Therefore, the current may be used to measure EMCD in magnetic materials [8].

The above relations motivate following probability current reconstruction based on two astigmatic transport of intensity (the continuity equation in an astigmatic paraxial optical system) (ATIE) [21] equations:

$$
\partial_{A_{1 n}} \rho(\mathbf{r})=-\frac{1}{k_{z}} \nabla_{\mathbf{e}_{n}} j_{n} \stackrel{\substack{\text { pure } \\ \text { state }}}{=}-\frac{1}{k_{z}} \nabla_{\mathbf{e}_{n}} \rho \nabla_{\mathbf{e}_{n}} \varphi
$$

where $\rho$ denotes the image intensity, $\varphi$ the wave's phase, $k_{z}=2 \pi / \lambda$ the wave number of the electrons, $A_{1 n}$ ( $\cong C_{20}, C_{02}$ in Ref. [21]) the strengths of the two line foci indexed by $n=\{0,1\}, \mathbf{e}_{n}$ the unit vector, and $j_{n}=\mathbf{e}_{n} \cdot \mathbf{j}$ the probability current perpendicular to the respective focal line. As the validity of the TIE is restricted to the quasimonochromatic case, where the use of an average wave number is permitted, large spectral widths with respect to the average electron energy, such as occurring with low beam energies must be avoided, e.g. by employing energy filters.

The left-hand side can be approximated by two slightly astigmatically defocused images as illustrated in Fig. $1[22,23]$. Instead of seeking a phase reconstruction (solving for the third term in Eq. (1), [21-24]), we, however, solve for the second term of this equation. Thereby, we are able to reconstruct both Cartesian components of the current

$$
\frac{\rho\left(\mathbf{r}, A_{1 n}\right)-\rho\left(\mathbf{r}, A_{1 n}+\delta A_{1 n}\right)}{\rho\left(\mathbf{r}, A_{1 n}\right)} \approx \delta A_{1 n} \rho\left(\mathbf{r}, A_{1 n}+\delta A_{1 n} / 2\right)
$$

instead of the curl-free (conservative) one obtained with one isotropic defocus (isotropic TIE) [25,26]. Moreover, we avoid a number of issues, mainly due to partial coherence and zeros in the wave field [26], limiting the conventional use of the TIE (typically with isotropic defocus) as a phase retrieval method. In particular, highresolution TIE phase or current retrievals from experimental data have not been previously reported to the best knowledge of the authors [27].

Integrating Eq. (1) under predefined boundary conditions (BCs) yields the components of the current density. For the following high-resolution study on a periodic crystal lattice we employ periodic BCs on the reconstructed area $\Omega$, permitting the following representation of the probability current as a Fourier series:

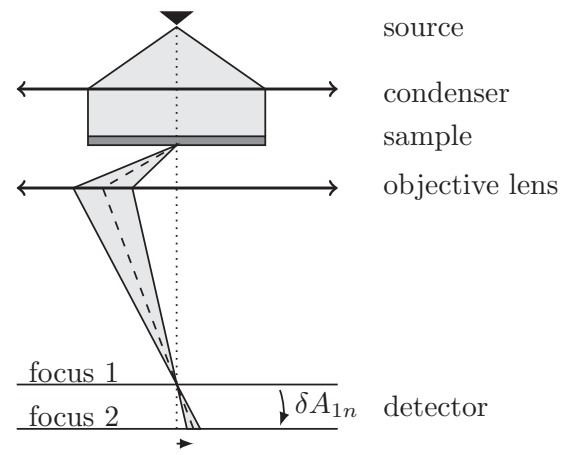

FIG. 1. 1D section of the optical setup for bright field TEM probability current reconstruction. The two-sided discrete approximation of the partial derivative with respect to a line focus is noted below. 


$$
j_{n}(\mathbf{r})=\sum_{\mathbf{k}} \tilde{j}_{n}(\mathbf{k}) e^{i \mathbf{k r}}
$$

with the Fourier coefficients

$$
\tilde{j}_{n}(\mathbf{k})=-i \frac{k_{z}}{k_{n} \Omega} \int_{\Omega} e^{-i \mathbf{k r}} \partial_{A_{1 n}} \rho(\mathbf{r}) d^{2} r .
$$

In order to obtain a well-defined Fourier series one has to apply some regularization at loci where $k_{n} \approx 0$ to suppress a large amplification of the inevitable experimental noise by dividing with $k_{n}$. In this work, we damp a small region around $k_{n}=\mathbf{e}_{n} \cdot \mathbf{k} \approx 0$ by multiplying with a suitable highpass filter [fil $=1-\exp \left(-k_{n}^{2} /\left(2 \sigma_{k n}^{2}\right)\right)$ with characteristic width $\sigma_{k n}$, see Fig. 2].

To obtain a good approximation of the differential line defocus on the left-hand side of Eq. (1) by a difference of two images, the astigmatic defocus $\delta A_{1 n}$ has to be in a range, where the corresponding Fresnel number is large (near field condition), i.e., $F=a^{2} /(\lambda \delta A) \gg 1$. Here, $a$ is the characteristic length to be resolved, i.e., the resolution of the TEM (approximately $0.1 \mathrm{~nm}$ in our case). We therefore require an astigmatic defocus in the range of a few nanometers, which is at the limit of adjustable focal step sizes in a state-of-the-art TEM. Note furthermore that the reconstructed probability current pertains to the image

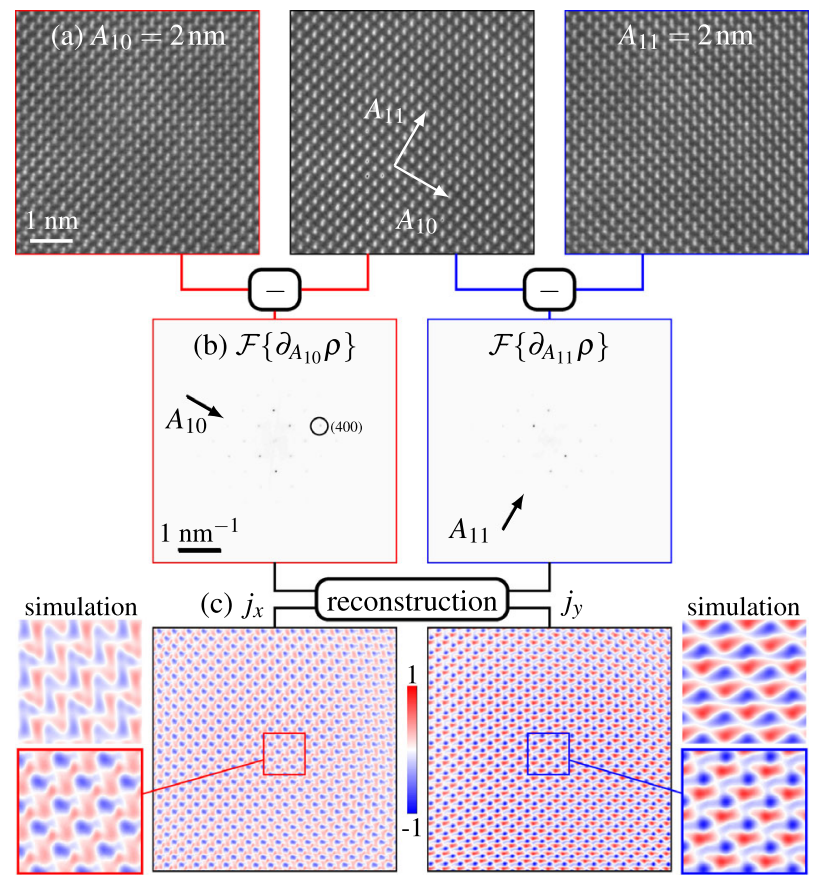

FIG. 2 (color online). Probability current reconstruction scheme. The astigmatic defocus series (a) is used to approximate the density derivatives (b), which are subsequently reconstructed following Eq. (3) to obtain the probability current (c). The maximal systematic reflection (004) used as resolution measure (approximately $0.1 \mathrm{~nm}$ ) is indicated. For visual purposes both intensity and probability current are scaled in arbitrary units. and not the object exit plane. Thus, aberrations of the optical system as well as the detector impair the original probability current at the object exit face, discussed above, similar to the conventional image intensity. In particular a convolution of the image intensity, e.g., due to the detector point spread function, carries over to the reconstructed probability current thereby reducing its spatial resolution, which follows directly from the vanishing commutation of convolution and derivation in Eq. (1).

We now demonstrate the crucial steps towards a probability current reconstruction at atomic resolution using a $\mathrm{SrTiO}_{3}$ sample thinned to about $10 \mathrm{~nm}$ in thickness [29]. For the experiments we used the Qu-Ant-TEM, a FEI Titan microscope aberration corrected both in the probe and imaging parts operating at $300 \mathrm{kV}$ (energy width $\sigma_{E}=0.8 \mathrm{eV}$, illumination semiconvergence angle $\sigma_{\theta}=0.15 \mathrm{mrad}$ ). All images were recorded on a Gatan Ultrascan US 1000XP CCD located under the viewing screen at a magnification of $640 \mathrm{kx}$ in Mh mode. Before acquiring the astigmatic defocus series, an image aberration free setting was adjusted $\left(A_{1}=1 \pm 1 \mathrm{~nm}, A_{2}=10 \pm\right.$ $20 \mathrm{~nm}, B_{2}=10 \pm 10 \mathrm{~nm}, C_{3}=-3 \pm 1 \mu \mathrm{m}, A_{3}<1 \mu \mathrm{m}$, $S_{3}<1 \mu \mathrm{m}$ using the standard aberration notation). A particularly large defocus of $C_{1}=148 \mathrm{~nm}$ has been deliberately chosen in order to simulate an incoherent image formation due to the corresponding incoherent envelope acting on the coherent image intensity $[30,31]$

$$
I_{\mathrm{img}}(\mathbf{r})=\frac{1}{\pi \sigma_{\theta}^{2} C_{1}^{2}} I_{\mathrm{coh}}(\mathbf{r}) * \exp \left(-\frac{1}{\sigma_{\theta}^{2} C_{1}^{2}} \mathbf{r}^{2}\right) .
$$

If we assume this incoherent envelope to be unknown (i.e., cannot be deconvolved from the recorded intensity), it in particular prevents phase reconstruction schemes requiring a well-defined coherent intensity. In the case of the probability current reconstruction, however, it only reduces the spatial resolution of the reconstructed current in the same way as the detector point spread does.

The crucial strengths of the two perpendicular objective stigmators of the microscope were quantified making use of the image hardware corrector as a calibrated measuring device. Moreover, the orientation of the corresponding stigmator axes was measured in the plane of the camera. The differential change of line defoci $\delta A_{1 n}=\delta C_{1} \pm \delta A_{1}$ is accomplished by a combined change of isotropic defocus (excitation of objective lens) and twofold astigmatism (excitation of column stigmators) by the same magnitude. By inspecting the observable image change we found that a defocus of $2 \mathrm{~nm}$ provided a good compromise between the high signal-to-noise ratio and large Fresnel number. In order to minimize any additional changes other than the line focus (e.g. specimen or aberration drift), we controlled the TEM by automated scripts allowing for a subsequent acquisition of slightly defocused images in a few seconds without any manual interference of the 
operator. Nevertheless, a drift of twofold astigmatism was observed upon recording the defocus series [32], which was taken into account when comparing the results to image simulations. Furthermore, an image registration step had to be applied before computing the difference between two images to remove the residual specimen drift. We used a memory-efficient subpixel registration cross-correlation based method optimized for large images [33,34]. The preprocessed series is depicted in Fig. 2(a).

The astigmatism change is readily revealed in the Fourier transform of the difference images [Fig. 2(b)] exhibiting a line of dampened reflections corresponding to the 1D filter $k_{n}$ in the direction of the calibrated astigmatism. Finally, the Fourier transformed probability current is obtained by inverting the filter and applying the regularization [Fig. 2(c)]. One readily observes some remaining noise in the low spatial frequency range, which might be further minimized by a stronger regularization (here $\sigma_{k n} \approx 1 / \mathrm{nm}$ ) at the expense of additional information loss.

The spatial resolution of the current densities as given by the highest order reflection in the Fourier transform amounts to approximately $1 \AA$ (see Fig. 2), which is equivalent to that of the underlying intensities. The most prominent feature in the reconstructed $x(y)$ components of the current density [see zoom-in in Fig. 2(c)] is a periodic arrangement of bright-dark contrast in the $x(y)$ direction located at the shoulders of the peaks of the corresponding conventional high-resolution intensity located at the $\mathrm{Sr}$ and $\mathrm{TiO}$ columns. This behavior is also observed in the corresponding STEM-DPC [16,17] and can be explained by the probability current converging to or diverging from the atomic columns depending on the respective dynamic scattering conditions. The enlarged vector plot in Fig. 3(b) underlines this behavior, in particular the converging current to the atomic columns. Moreover, the decomposition into conservative [Fig. 3(a)] and solenoidal components [Fig. 3(c)] reveals a significant solenoidal current between the columns, which we mainly attribute to the symmetry breaking induced by the previously mentioned twofold astigmatism of $6 \mathrm{~nm}$ (orientation: $17^{\circ}$ ), which has developed while recording the defocus series.

To verify the accuracy of the reconstruction we performed two tests, an independent TIE reconstruction from an isotropic defocus and numerical scattering and imaging simulations of the probability current (and intensities) taking into account the residual geometric aberrations, the spatial and chromatic incoherence, as well as the camera point spread. In particular, the conservative current pertaining to the isotropic defocus [Fig. 3(d)] agrees very well with the one obtained from the Helmholtz decomposition, which corroborates that the two line defoci have been well adjusted in both direction and magnitude. Also, the numerical results [Fig. 3(e)] fit well after refining the sample thickness and taking into account a twofold astigmatism of $6 \mathrm{~nm}$. We attribute the remaining

(a) $\mathbf{j}_{\mathrm{c}}$ aTIE

(b) $\rho+\mathbf{j}$ aTIE

(c) $2 \times \mathbf{j}_{\mathrm{r}} \quad$ aTIE
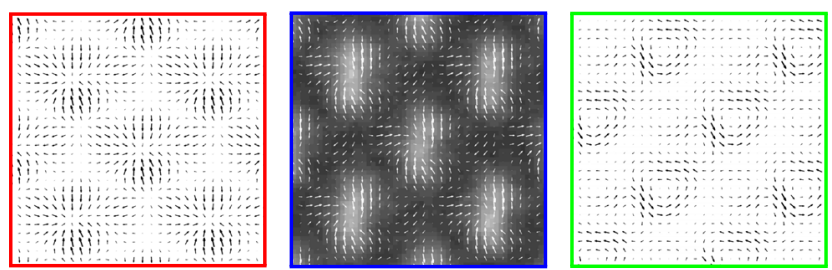

(d) $\quad \mathbf{j}_{\mathrm{c}}$ iTIE

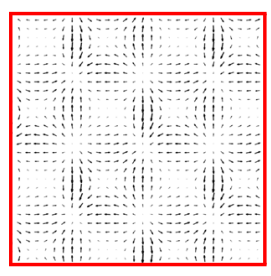

(e) $\rho+\mathbf{j} \operatorname{sim}$

(f)
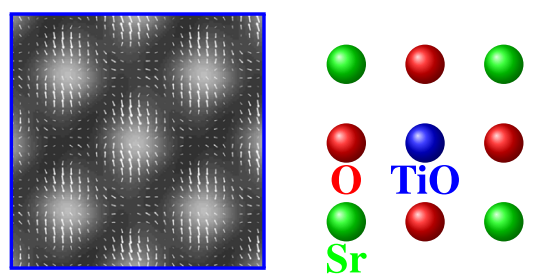

FIG. 3 (color online). Averaged conservative (a), total (b), and two-times-amplified solenoidal probability current (c) reconstructed from the astigmatic defocus series over one unit cell of $\mathrm{SrTiO}_{3}$ (f). The conservative current obtained from an isotropic defocus reconstruction (d) as well as the complete current obtained from a scattering simulation (e) are given for comparison. The corresponding gray scaled intensity images are depicted in the background of (b) and (e).

discrepancies to a slight sample misalignment and the errors of the experimentally determined or numerical fitted simulation parameters, such as defocus, sample thickness, residual aberrations, orientation of the line foci, anisotropic magnification, etc.

In summary, we have demonstrated the reconstruction of the probability current at atomic resolution over large fields of view. The crucial steps are the acquisition of an astigmatic defocal series, an accurate alignment of the micrographs, and a regularization of the reconstruction procedure. We see the prospects of the current reconstruction mainly in intrinsically incoherent imaging regimes. The technique can be used to study elastic scattering from electric, magnetic, and strain fields, down to atomic resolution, with incoherent electron beams such as employed in ultrafast TEM, where otherwise very accurate coherent phase retrieval techniques such as offaxis electron holography (e.g., Ref. [35]) fail. Even more importantly, the atomically resolved probability current can be recorded through an energy filter, where it exposes particular characteristics of the core-loss inelastic transition not visible in a conventional electron energy loss spectrum (EELS) or EFTEM experiment. Such a measurement could detect the magnetic information from the solenoidal part of the probability current which can be seen as a variant of the conventional EMCD technique [1]. Similar spectroscopic setups can also work at medium scale resolution. Other possible applications include strain mapping and band structure evaluation exploiting the link between the probability current and these sample properties. 
J. V. and A. B. acknowledge funding from the European Research Council under the 7th Framework Program (FP7), ERC Starting Grant No. 278510 VORTEX. The Qu-AntEM microscope was partly funded by the Hercules fund from the Flemish Government. All authors acknowledge financial support from the European Union under the Seventh Framework Program under a contract for an Integrated Infrastructure Initiative. Reference No. 312483ESTEEM2. J. V. acknowledges funding from the FWO under Project No. G.0044.13N.

[1] P. Schattschneider, S. Rubino, C. Hebert, J. Rusz, J. Kunes, P. Novák, E. Carlino, M. Fabrizioli, G. Panaccione, and G. Rossi, Nature (London) 441, 486 (2006).

[2] Introduction to Electron Holography edited by E. Völkl, L. Allard, and D. C. Joy (Kluwer Academic/Plenum Publishers, New York, 1999)

[3] H. S. Park, X. Yu, S. Aizawa, T. Tanigaki, T. Akashi, Y. Takahashi, T. Matsuda, N. Kanazawa, Y. Onose, D. Shindo, A. Tonomura, and Y. Tokura, Nat. Nanotechnol. 9, 337 (2014).

[4] M. Hytch, F. Houdellier, F. Hue, and E. Snoeck, Nature (London) 453, 1086 (2008).

[5] C. T. Koch, V. B. Özdöl, and P. A. van Aken, Appl. Phys. Lett. 96, 091901 (2010).

[6] Scanning Transmission Electron Microscopy edited by N. P. D. Pennycook and J. Stephen (Springer, New York, 2011).

[7] J. Rusz, J. C. Idrobo, and S. Bhowmick, Phys. Rev. Lett. 113, 145501 (2014).

[8] P. Schattschneider, B. Schaffer, I. Ennen, and J. Verbeeck, Phys. Rev. B 85, 134422 (2012).

[9] This does not contradict the uncertainty relation as the current is reconstructed from a set of images.

[10] B. Barwick, D. J. Flannigan, and A. H. Zewail, Nature (London) 462, 902 (2009).

[11] O.-H. Kwon and A. H. Zewail, Science 328, 1668 (2010).

[12] D. J. Flannigan and A. H. Zewail, Acc. Chem. Res. 45, 1828 (2012).

[13] H. Rose, Optik (Stuttgart) 39, 416 (1973).
[14] N. H. Dekkers and H. de Lang, Optik (Stuttgart) 41, 452 (1974).

[15] A. Lubk and J. Zweck, Phys. Rev. A 91, 023805 (2015).

[16] N. Shibata, S. D. Findlay, Y. Kohno, H. Sawada, Y. Kondo, and Y. Ikuhara, Nat. Phys. 8, 611 (2012).

[17] K. Müller, F. F. Krause, A. Béché, M. Schowalter, V. Galioit, S. Löffler, J. Verbeeck, J. Zweck, P. Schattschneider, and A. Rosenauer, Nat. Commun. 5, 5653 (2014).

[18] N. Shibata, Y. Kohno, S. D. Findlay, H. Sawada, Y. Kondo, and Y. Ikuhara, J. Electron Microsc. 59, 473 (2010).

[19] D. Paganin and K. A. Nugent, Phys. Rev. Lett. 80, 2586 (1998).

[20] L. Peng, S. Dudarev, and J. Whelan, High Energy Electron Diffraction and Microscopy, Monographs on the Physics and Chemistry of Materials (Oxford University Press, New York, 2004).

[21] L. J. Allen, M. P. Oxley, and D. Paganin, Phys. Rev. Lett. 87, 123902 (2001).

[22] W. McBride, N. L. O'Leary, K. A. Nugent, and L. J. Allen, Acta Crystallogr. Sect. A 61, 321 (2005).

[23] T. C. Petersen and V. J. Keast, Ultramicroscopy 107, 635 (2007).

[24] C. A. Henderson, G. J. Williams, A. G. Peele, H. M. Quiney, and K. A. Nugent, Opt. Express 17, 11905 (2009).

[25] M. R. Teague, J. Opt. Soc. Am. 73, 1434 (1983).

[26] A. Lubk, G. Guzzinati, F. Börrnert, and J. Verbeeck, Phys. Rev. Lett. 111, 173902 (2013).

[27] The phase reconstruction reported by Ishizuka and Allman [28] pertains to the curl-free probability current instead of the wave.

[28] K. Ishizuka and B. Allman, J. Electron Microsc. 54, 191 (2005).

[29] A value of $10.9 \mathrm{~nm}$ yielded a maximal agreement with image simulations.

[30] A. Lubk and F. Röder, Ultramicroscopy 151, 136 (2015).

[31] C. T. Koch, Ultramicroscopy 108, 141 (2008).

[32] J. Barthel and A. Thust, Ultramicroscopy 134, 6 (2013).

[33] J. R. Fienup and A. M. Kowalczyk, J. Opt. Soc. Am. A 7, 450 (1990).

[34] M. Guizar-Sicairos, S. T. Thurman, and J. R. Fienup, Opt. Lett. 33, 156 (2008).

[35] F. Genz, T. Niermann, B. Buijsse, B. Freitag, and M. Lehmann, Ultramicroscopy 147, 33 (2014). 